Published in final edited form as:

Methods Enzymol. 2015 ; 564: 501-527. doi:10.1016/bs.mie.2015.08.017.

\title{
In vivo $\mathrm{pO}_{2}$ imaging of tumors: Oxymetry with very low frequency Electron Paramagnetic Resonance
}

\author{
Boris Epel and Howard J. Halpern \\ Center for Electron Paramagnetic Resonance Imaging In Vivo Physiology, Department of \\ Radiation and Cellular Oncology, University of Chicago, Chicago, IL
}

\begin{abstract}
For over a century it has been known that tumor hypoxia, regions of a tumor with low levels of oxygenation, are important contributors to tumor resistance to radiation therapy and failure of radiation treatment of cancer. Recently, using novel pulse electron paramagnetic resonance (EPR) oxygen imaging, near absolute images of the partial pressure of oxygen $\left(\mathrm{pO}_{2}\right)$ in tumors of living animals have been obtained. We discuss here the means by which EPR signals can be obtained in living tissues and tumors. We review development of EPR methods to image the $\mathrm{pO}_{2}$ in tumors and the potential for the $\mathrm{pO}_{2}$ image acquisition in human subjects.
\end{abstract}

\section{Introduction: The importance of imaging molecular oxygen in cancer therapy}

A near universal characteristic of solid human tumors is hypoxia. Hypoxia is defined as clinically significant regions of a malignant tumor with low levels of molecular oxygen, or low values of $\mathrm{pO}_{2}$. Resistance of human solid tumors to radiation induced by such regions of hypoxia has been recognized for over a century. ${ }^{1}$ Similar resistance has more recently been noted for chemotherapy. ${ }^{2,3}$ Human trials investigating hyperbaric oxygen and hypoxic sensitizers to overcome tumor hypoxia have shown promise but limited success. ${ }^{4,5}$ However, the extensive variability of tumor oxygenation, both in overall extent and in the location of hypoxic regions, may confound such attempts. The results demonstrate the importance of, assessment not only of overall tumor oxygenation, but of locating, through imaging, significant regions of hypoxia in the tumor. We will briefly review this data, and then discuss various alternative methods of measuring tissue and imaging tumor oxygenation.

\section{Imaging Physiology in Living Animals}

Short of transillumination, the first images of deep structures in living animals began with Wilhelm Roentgen's discovery of the X-ray in $1895^{6}$ Since then imaging modalities have evolved sensitivity to varied soft tissue states of animals and humans. ${ }^{7,8}$ In parallel, spectroscopies have evolved, revealing the physical aspects of solid and liquid states of matter. These states are the environment in which biologic processes evolve. ${ }^{9}$ However, living samples are heterogeneous. The full definition of physiologic states, thus, requires spectroscopic imaging. Various forms of spectroscopic imaging have evolved in the past 
three decades. This makes available from the spectroscopic process information that localized within a subvolume of a living sample. ${ }^{10,11}$

\section{The Nature of Molecular Oxygen}

The oxygen molecule, $\mathrm{O}_{2}$, is a diradical with two unpaired electrons in the triplet state that define its interaction with other molecules bearing unpaired electron and nuclear spins and with electromagnetic radiation. It is a rapidly tumbling diatomic molecule with its two unpaired spins rapidly relaxing each other. Thus, the oxygen molecule in solution at room temperature has a nearly unmeasurably fast electron relaxation rate. When interacting with another molecule bearing a single highly stable unpaired electron spin-- a spin probe-oxygen increases the relaxation rates of the probe, mainly via Heisenberg exchange ${ }^{12,13}$. The solution interaction rates between the spin probe and $\mathrm{O}_{2}$ are described by the Smoluchowski diffusion equation, which predicts a linear relation between $\mathrm{pO}_{2}$ and spin probe relaxation rate, validated for multiple free radicals ${ }^{14}$.

\section{Measurement of Molecular Oxygen}

A number of techniques for measurement of molecular oxygen have been developed. The chemical and physical properties of oxygen enable a variety of methods, each with their own applicability and advantages. The "gold standard" of oxymetry in cells and live animal tissues is the platinum electrode. ${ }^{15} \mathrm{~A}$ recent enhancement of the standard platinum electrode is the Eppendorf electrode. This is inserted into tissues of living animals and human subjects with a highly regular advance and retreat pattern for measurement consistency. It has a 200$300 \mu \mathrm{m}$ diameter tip that is inserted into the tissue, which measures oxygen along a series of tracks ${ }^{16}$. The OxyLite ${ }^{\mathrm{TM}}$ probe (Oxford Optronix, Oxford, UK) utilizes fluorescence quenching of fluorophore by oxygen ${ }^{17}$. OxyLite ${ }^{\mathrm{TM}}$ can be used for repetitive measurements in the same location or along tracks, although the $200 \mu \mathrm{m}$ glass fiber will wander from a straight line sampling. The electrode and Oxylite provide highly local samples, and do not provide an overall inventory of the $\mathrm{pO}_{2}$ distributions in tissues.

There are a number of noninvasive qualitative oxymetries available. These include nearinfrared spectroscopy, typically of blood saturation, fluoramisonidozole retention $\mathrm{PET}^{18}$, and Blood Oxygenation Level Dependent (BOLD) Magnetic Resonance Imaging $(\mathrm{MRI})^{19,20}$. These methods are subject to confounding variation that frustrates quantitative measurement, and, particularly if the animal is stressed or otherwise changes its state, prevents reliable repeated measurement. Phosphorescence quenching ${ }^{21},{ }^{19} \mathrm{~F}^{22,23}$ protonelectron double resonance imaging (PEDRI) ${ }^{19,24}$ and Electron Paramagnetic Resonance (EPR) are more quantitative $\mathrm{pO}_{2}$ imaging modalities. Despite their quantitative imaging capabilities, phosphorescence quenching, ${ }^{19} \mathrm{~F}$ MRI ${ }^{20}$ and PEDRI are still subject to confounding variation, most significantly from the effect of the concentration of the sensing molecule (the phosphorescent compound, the ${ }^{19} \mathrm{~F}$ for MRI, or the self relaxation of the electron bearing spin probe and the relaxation time of the water proton in PEDRI) on the relaxation rate-dependence on $\mathrm{O}_{2}$. Solutions to this problem have included the use of EPR with particulates or application of longitudinal or spin lattice relaxation rate EPR imaging of 
soluble spin probes, that provide near absolute measurements of local $\mathrm{pO}_{2}$, with order-ofmagnitude reduction in confounding variation.

\section{Principles of Electron Paramagnetic Resonance}

The Electron Paramagnetic Resonance technique detects molecular species with one or more unpaired electrons: paramagnetic complexes, radicals, lattice defects, etc. Principles of magnetic resonance are covered in a number of books ${ }^{25-28}$. Here, we discuss essential relevant aspects of the technique for understanding EPR imaging and oxymetry.

An unpaired electron possesses an intrinsic magnetic moment not associated with its orbital angular momentum. The spin moment is $m=-\frac{1}{2 \hbar} g \mu_{B}$ where $g$ is the electron $g$-factor equal to 2.0023 for a free electron, $\mu_{B}$ is the Bohr electron magneton and $\hbar$ is Planck's constant divided by $2 \pi$.

A crucial difference between the technique of electron paramagnetic resonance and that of nuclear magnetic resonance and their respective imaging techniques is the difference in the magnitude of the magnetic moments. This three order of magnitude difference completely transforms the respective methods. Relativistic quantum mechanics predicts the spin magnetic moment $\mu_{\mathrm{B}}$ to be, $\mu_{B}=\hbar e / 2 \pi m c^{29}$, where $e$ is the magnitude of the charge of the electron or proton, $c$ is the speed of light and $m$ is the mass of either the electron or, in the case of a hydrogen nucleus, the mass of the proton. The magnetic moment of the electron is 1836 times larger than that of the proton. Due to the anomalous magnetic moment of the proton, which is 2.79 times larger than the pure relativistic prediction because of the finite size or non-point charge distribution of the proton, the actual magnetic moment of the electron is 658 times or still nearly three orders of magnitude larger than that of the proton.

In the presence of a static magnetic field $\left(B_{0}\right)$, the energy $(E)$ of a magnetic moment depends on its orientation relative to $B_{0}$. For a spin $1 / 2$ particle $(S=1 / 2)$, quantum mechanics counterintuitively requires orientations to be parallel (-) or anti-parallel (+) to the imposed magnetic field $B_{0}$.

$$
E= \pm \frac{1}{2} g \mu_{B} B_{0} \quad[1]
$$

The difference between these energy levels $(\Delta E)$ can be described in terms of angular frequency $\left(\omega_{0}\right)$ via Planck's energy-frequency relationship.

$$
\Delta E=\hbar \omega_{0} \quad[2]
$$

Combining these two equations gives the relation between frequency and applied field as:

$$
\omega_{0}=\gamma_{e} B_{0} \quad[3]
$$

where $\gamma_{\mathrm{e}}=g \mu_{\mathrm{B}} / \hbar$ is the electron gyromagnetic ratio. This is equal to $1.76 \times 10^{11} \mathrm{~s}^{-1} \mathrm{~T}^{-1}$ for the free electron. The three order of magnitude smaller proton gyromagnetic ratio $\gamma_{p}$ is 2.68 
$\times 10^{8} \mathrm{~s}^{-1} \mathrm{~T}^{-1} \cdot \omega_{0}$ is known as the Larmor frequency, the Zeeman frequency or the basic resonance frequency.

The major instrumental consequences of these results are

1. The magnetic field at which an electron magnetic absorbs significantly more energy from the oscillating electromagnetic field -- resonance -- is approximately 658 times smaller $\left(=\gamma_{\mathrm{p}} / \gamma_{\mathrm{e}}\right)$ than that for a proton at a given excitation frequency, $\omega_{0}$. The "approximately" is due to the inevitable modification of the local environment of the resonating electron by the magnetic fields of nearby nuclei or, rarely, unpaired electrons. This means that at high field MRI frequencies, EPR imagers use much lower magnetic fields.

2. The rates at which excited electron spins relax are proportional to $\gamma^{2} \cdot{ }^{30}$ Electrons relax $\sim\left(658\left(=\gamma_{\mathrm{e}} / \gamma_{\mathrm{p}}\right)\right)^{2}$ or nearly six orders of magnitude faster than water protons. Electrons relax in times of nanoseconds to microseconds. Water protons and common nuclei relax in tens of milliseconds to many tens of seconds. Excited ${ }^{13} \mathrm{C}$ enriched pyruvate, for example in human subjects, relaxes in $\sim 1$ minute.

The numbers of unpaired spins in common samples measured with magnetic resonance, either electron spins or nuclear spins, are very large, from a billion billion to a trillion trillion spins, which are thermodynamic numbers. These are subject to thermodynamic energy distributions, the Boltzmann distribution, with smaller numbers of spins in the higher energy orientation than in the lower energy orientation. The magnitude of the equilibrium magnetization is proportional to the imposed magnetic field, $B_{0}$. The overall magnetic moment summed over all the magnetic moments of the spins is referred to as the magnetization of the spin system. The magnetization in a sample can be manipulated by addition of a second, oscillating magnetic field $\left(B_{1}\right)$. To be effective for $\mathrm{S}=1 / 2$ states, $B_{1}$ is oriented orthogonal to $B_{0}$ and oscillates at frequencies close to the Larmor frequency. The time varying magnetic field $B_{1}$ can add energy to the magnetization system. It accomplishes this by inverting the orientations of individual electronic spins and changing the direction of the overall sum of the unpaired spins, the magnetization. The phenomenological Bloch equation describes the time evolution of magnetization orientation in the presence of both $B_{0}$ and $B_{1}$ magnetic fields ${ }^{25}$. In a coordinate system rotating at the spectrometer operating frequency where $B_{1}$ is not changing, the return of transverse $\left(M_{T}\right)$ and longitudinal $\left(M_{Z}\right)$ components of magnetization to equilibrium in the absence of $B_{1}$ is described by ${ }^{25}$ :

$$
\begin{gathered}
M_{T}(t) \propto \exp (i \Omega t) \exp \left(-\frac{t}{T_{2}}\right) \\
M_{Z}(t) \propto\left(1-\mathrm{M} \cdot \exp \left(-\frac{t}{T_{1}}\right)\right)
\end{gathered}
$$

Here, $\Omega$ is the difference between the Larmor frequency of the electron and the EPR spectrometer's operating frequency. The longitudinal relaxtion time, $T_{1}$, and the transverse relaxation time, $T_{2}$, correspond to the relaxation of $M_{T}$ and $M_{Z}$, respectively. $T_{1}$ and $T_{2}$ are 
the inverses of the respective longitudinal relaxation rate, $R_{1}$, and transverse relaxation rate, $R_{2}$. The conventional EPR time domain signal, $s(t)$, is proportional to $M_{T} . M$ describes an initial state of longitudinal magnetization. The longitudinal magnetization can be encoded into the EPR signal by using special pulse sequences. The transverse magnetization relaxation rate, $\mathrm{R}_{2}$, is commonly referred to as the phase memory relaxation rate. This is the rate at which spins that have been aligned by a very short duration pulse of oscillating magnetic field, which prepares them in a particular magnetization state, lose their coherent alignment in the time after the pulse.

The first EPR experiments were continuous wave (CW) EPR experiments. Here the $B_{1}$ excitation is applied continuously during an experiment or image. In a $\mathrm{CW}$ spectrometer, the EPR signal is produced by applying $B_{1}$ at a fixed frequency $\omega_{0}$ and sweeping $B_{0}$ through the resonance condition of Equation [3]. ${ }^{28}$ In principle, the field $B_{0}$ could be fixed and the frequency swept through the resonance condition of Equation [3], but the former approach simplifies the electronics and allows a simpler amplification of signal. An alternate strategy applied often to simpler, more robust systems, involves subjecting the unpaired electron spin-bearing sample, prepared in a magnetic field, to a high power pulse of radiation, of duration shorter than $T_{2}$. This pulse aligns the magnetization in the magnetic field. Detecting the rate of decay of the magnetization signal is the basis of this latter experiment. This is referred to as a time domain experiment. It is important to note that the $\mathrm{CW}$ magnetic field domain spectrum is related to the time domain signal through a Fourier transformation.

$$
S(\mathrm{~B})=\int_{-\infty}^{\infty} s(t) \exp \left(-i \gamma_{e} \mathrm{~B} t\right) d t \quad[6]
$$

Note that the factor $\gamma_{\mathrm{e}}$ converts magnetic field units into inverse time, or rate.

\section{Oxymetry with EPR}

Injectable, water soluble spin label or spin probe EPR oxymetry is a minimally invasive method that can report absolute $\mathrm{pO}_{2}$ deep in tissues ${ }^{31-33}$. In the 1980's, EPR detection of oxygen using the broadening of the width of the EPR spectrum from a nitroxide spin probe was first reported by Backer et al. ${ }^{34}$ and Popp et al. ${ }^{35}$ and later extensively investigated by Swartz and coworkers ${ }^{14,33,36}$, by using various classes of spin probes. In the nineties, a few groups pioneered multi-dimensional $\mathrm{CW}$ imaging on rodents in vivo and ex vivo, enabling repeated measurements of oxygen concentrations in living tissues ${ }^{37-39}$.

The underlying mechanism of interaction between spin probe and oxygen is predominantly Heisenberg spin exchange. ${ }^{29}$ In this mechanism, the electrons from the rapidly relaxing oxygen environment, during the encounter with a spin probe, are not distinguishable from the electrons of the spin probe. The spin probe electrons thereby share their environment with that of oxygen during which time they relax more rapidly. The increased rate loss of both phase and energy of the spin probe electron is proportional to the rate of encounter with oxygen. This is directly proportional to the oxygen concentration and the oxygen partial pressure. 
EPR techniques like CW or time domain free induction decay (FID) pulse imaging, measure total EPR decay rates or line widths (LW) instead of the transverse or longitudinal relaxation rates. The total EPR decay rate/line width of free radicals is due to two major components: 1) homogeneous broadening due to transverse relaxation and 2) inhomogeneous broadening due to the interaction of the electron with neighboring paramagnetic nuclei ${ }^{26,27}$. In the case of inhomogeneous broadening, the electron experiences the magnetic fields of multiple neighboring nuclei, which variably shifts the resonance frequency, creating hyperfine (HF) spectral structure. A more precise method for obtaining spin packet line width, that is used in continuous wave oxymetry, explicitly fits the EPR line to spectral models that include hyperfine structure, and accounts for the effect of the magnetic field modulation ${ }^{40-42}$.

In addition to partial pressure of molecular oxygen, details of EPR lineshape provide an insite into the thiol-disulfide balance or redox state, the related local bioreduction capability, the acidity $(\mathrm{pH})$, the temperature, the presence of specific oxygen centered free radicals, and many others. ${ }^{43}$

Characterizing the spin relaxation by relaxation rates is the inverse of characterizing it by relaxation times. Relaxation rates are related to relaxation times, $T: R=1 / T$. Relaxation rates can be multiplied by $\left(\gamma_{\mathrm{e}}\right)^{-1}$, the inverse of the gyromagnetic ratio, to allow expression of the rates directly in magnetic field units $(\mu \mathrm{T})$. Thus, line broadening is directly related to relaxation rate increase. Relaxation rates naturally demonstrate the relationship between increasing numbers of relaxation mechanisms and increasing relaxation rates, or linewidths. This process also provides a conceptual link between parameters determined by CW and pulse methods. For Lorentzian line shapes, the half width at half maximum (HWHM) is equal to $1 /\left(\gamma_{\mathrm{e}} T_{2}\right)=\left(1 / \gamma_{\mathrm{e}}\right) R_{2}$. For EPR lines with multiple broadening mechanisms, $\left(1 / \gamma_{\mathrm{e}}\right) R_{2}$ describes the homogeneous broadening of the EPR line (spin packet line width). In early $\mathrm{CW}$ oxymetry, the spin packet line width allowed the determination of $\mathrm{pO}_{2}$.

Transverse relaxation-based oxymetry is more susceptible to variation of other physical parameters beside oxygen partial pressure. Parameters such as temperature, viscosity and salinity are tightly controlled in the body of a living animal ${ }^{44}$. This allows a quantitative enumeration of their effects on relaxation, allowing correction and calibration of oxygen measurements. However, spin probe concentration may vary and affect the accuracy of line width/transverse relaxation rate-based oxymetry.

\section{Spin Probe Sensitivity to Molecular Oxygen $\left(\mathrm{O}_{2}\right.$ or $\left.\mathrm{pO}_{2}\right)$ and Other Environmental Factors}

Heisenberg spin exchange between a spin probe and oxygen acts on the spin probe's

longitudinal relaxation in a manner nearly identical to action on transverse relaxation ${ }^{13,45}$. Importantly, other relaxation processes affect transverse and longitudinal relaxation differently. Electron spin exchange between two trityl spin probe molecules increases $R_{2}{ }^{13}$. The effect of the spin exchange between spin probes on $R_{2}$ is dependent on the number of collisions per unit time, and, therefore, on the concentration of spin probe. $R_{2}$ cannot distinguish between the dephasing effect of an interaction of a spin probe with oxygen and a dephasing effect of an interaction of a spin probe with another spin probe. The exchange of 
energies between two spin probes, however, does not alter the total energy of the interacting spin pair, and therefore does not affect the spin probe longitudinal magnetization component. Thus, $R_{1}$ is much less susceptible to self-broadening. $R_{1}$ is nearly an absolute measure of oxygen concentration, or $\mathrm{pO}_{2}$. Fig. 2 shows the effect of $\mathrm{O}_{2}$ concentration, or $\mathrm{pO}_{2}$, on the relaxation rates, $R_{1}$ and $R_{2}$, to have identical slopes, but there is nearly an order of magnitude reduction in the dependence of $R_{1}$ on spin probe concentration relative to $R_{2}$. $R_{1}$ is a near absolute measure of $\mathrm{pO}_{2}$ with precision exceeding 1 torr. This increase in probe specificity is a distinguishing feature of EPR oxymetry, in comparison with other noninvasive methods ${ }^{32}$.

The ability of an EPR image to quantify molecular oxygen dissolved in the life supporting solvents of a living animal and eventually a human is among the most important of its abilities. The absence of oxygen in the heart, brain, and limbs affected by peripheral vascular disease or diabetes, ischemic bowel, and portions of cancers of human patients rendered resistant to therapy by the absence of oxygen, is responsible for the death of greater than half of our species. The evaluation of treatments and pharmaceutical agents to ameliorate the hypoxic state promises to prolong the useful and involved lives of all of us. ${ }^{46}$

\section{Radio frequency Magnetization Excitation is Necessary for In Vivo EPR Imaging of Large Animals and Humans}

EPR imaging needs to be done under unusual conditions for eventual human application. Conventional EPR spectroscopy is usually carried out at frequencies of gigahertz to hundreds of gigahertz. Lower frequencies are required to penetrate deeply into the body of a human composed two thirds by weigh of salt water. The non-resonant loss of signal amplitude caused by conductive loss at low frequency, or dipolar loss at higher frequency, requires magnetization excitation frequencies of the order of hundreds of $\mathrm{MHz}$ for large human-size animals. This is the excitation frequency of a high field, whole body MRI. For small animals, frequencies of up to approximately $1 \mathrm{GHz}$ can be used. ${ }^{47,48}$ As we mention above, unlike MRI, which requires multi-Tesla superconducting magnets, at $250 \mathrm{MHz}$, the $B_{0}$ for EPR images is 9 milliTesla. These stationary magnetic fields can be generated by simple copper air core magnets. Small animal experiments to rapidly and efficiently evaluate treatment and pharmaceutical effectiveness, may use higher frequencies near $1 \mathrm{GHz}$ (Lband), that will give higher signals and will not suffer paralyzing loss that would make large animal experiments difficult. Because relaxation rates of even the most slowly relaxing aqueous spin probes are five to six orders of magnitude faster than those of a water hydrogen nucleus, fixed stepped gradients, tomographic image reconstruction and modest power requirements make the technique relatively inexpensive.

\section{Dissolved Spin Probe Oxymetry}

In general, quantification of aspects of the tissue microenvironment requires an environmental reporter and a readout technique. The reporter can be endogenous, such as water protons or sodium ions in MRI, or exogenous, such as implanted particulates or injected soluble spin probes. Endogenous reporters typically have much higher concentrations than exogenous ones and therefore, are easier to detect, but their localization 
cannot be controlled. Consequently, they are non-specific and sometimes provide an overwhelming background signal that mask a signal of interest. On the contrary, exogenous spin probes can be specifically targeted to the areas of interest.

Endogenous paramagnetic species found in mammalian bodies include hemoglobin, metalloenzymes, but extremely low concentrations of unbound diffusible species except molecular oxygen. Metal centers and oxygen at animal body temperature have very short relaxation times, broad lines and thus are not easily and directly measurable at low EPR frequencies. Moreover, unbound diffusible paramagnetic metal species, can interact with and transform covalent carbon-carbon and carbon-hydrogen molecular bonds as they do as enzymatic reaction centers. Therefore, living systems have developed arrays of binding proteins to maintain endogenous diffusible concentrations below 1 nanomole because of this threat to covalent bonds of the molecules of living systems. At present, exogenous spin probes are really the only practical reporters, and appropriate spin probes are the key to successful imaging. The line width and relaxation times of the probe, and their sensitivity to oxygen, largely define sensitivity of methodology and $\mathrm{pO}_{2}$ accuracy. Probes with narrower line widths and longer relaxation times allow higher resolution imaging. High fractional sensitivity of line width to oxygen ensures better imaging accuracy. Finally, the spin probes should be minimally toxic and metabolically stable.

Summarizing the above, EPR oxymetric imaging requires a spin probe to sample the fluid environment and report the oxygen partial pressure through increase in its spin packet line width or, equivalently, its relaxation rates. Measurement of line broadening of nitroxide spin probes has, until recently, been the principle means of dissolved spin probe oxymetry. Hypoxic nitroxide relaxation rates, $1-2 \mu \mathrm{s}^{-1}$, are still too rapid for imaging at several hundred MHz excitation frequencies. Tri-aryl methyl radicals, specifically, partiallydeuterated methyl-tris[8-carboxy-2,2,6,6-tetrakis[2-hydroxyethyl]benzo[1,2-d:4,5d']bis[1,3]dithiol-4-yl]-trisodium salt, OX063, have an order of magnitude smaller hypoxic $R_{1}$ value of $1 / 6 \mu \mathrm{s}^{-1}\left(T_{1}=6 \mu \mathrm{s}\right)$ These relaxation rates enable pulse measurement and imaging at $250 \mathrm{MHz} .{ }^{49} R_{1}$, measured with inversion recovery pulse sequences, increases signal to noise by nearly a factor of two relative to electron spin echo imaging, which measures $R_{2}$, and has reduced the confounding sensitivity of spin probe to the self-relaxation or broadening described above, by nearly an order of magnitude. This makes the spin probe measurement accurate to within 1-2 torr, an absolute measurement or image for animal application (vide infra). ${ }^{50}$

At present, two large classes of spin probes are used for in vivo oxymetry: Soluble free radicals and insoluble paramagnetic particles (particulates) ${ }^{51,52}$. They are introduced into animals in different ways and require different imaging methods.

\section{Soluble spin probes}

Physiologic EPR imaging has been enabled by the synthesis of free radical reporter molecules. These molecules distribute in specific physiologic compartments. Desirable characteristics for these probes include water solubility, kinetic and metabolic stability, a single narrow line resonance, line widths or relaxation rates directly related to $\mathrm{pO}_{2}$, 
persistence of signal from tumors longer than imaging times, and low toxicity 32 .

Historically, the first probes applied for oxymetry were nitroxides (Figure 1A) $53,54,55,56$.

\section{Trityls}

The recent success of spin probe oxymetry was enabled by triarylmethyl radicals (TAM radicals), which are also referred to as trityls (Figure 1B). Trityl compounds were developed by Nycomed innovation (later acquired by GE Healthcare; Little Chalfont, Buckinghamshire, United Kingdom). Trityls have extremely narrow, single EPR lines ${ }^{55}$. Those which are used for in vivo imaging are OX063 (16 $\mu \mathrm{T}$ peak-to-peak, p-p) and its partially deuterated form $\mathrm{Ox}_{63} \mathrm{~d}_{24}(8 \mu \mathrm{T} \mathrm{p}-\mathrm{p})$ (Figure 1B). The trityl $R_{1}$ and $R_{2}$ is linearly dependent on $\mathrm{pO}_{2} 55$ (Figure 2). These molecules are triacid, charge 3- anionic and distribute in the extracellular fluid compartment ${ }^{32,57}$. In the blood stream of a mouse, the clearance halftime of these probes is $9-10 \mathrm{~min}$, while in tumors they remain and provide strong signals for $40-50 \mathrm{~min}^{58}$. The dose at which $50 \%$ of animals die, the $\mathrm{LD}_{50}$, of OX063 is large $(8 \mathrm{mmol} / \mathrm{kg})$, which allows high dose injections ${ }^{59}$. Typically, $0.5 \mathrm{ml}$ of 80 to $100 \mathrm{mM}$ solutions at neutral $\mathrm{pH}$ are continuously injected intravenously (IV) into 20-25g animals to give tumor average concentrations of several hundred $\mu \mathrm{M}$.

\section{Particulates}

A number of different solid, crystalline particulates have been used for EPR oxymetry: Activated charcoal, and lithium phthalocyanine ${ }^{60}$ and its derivatives with higher oxygen sensitivity, such as octa- $n$-butoxy-naphthalocyanine ${ }^{56}$ (Figure 1C). These insoluble particulate spin probes can be inserted surgically as several tens or hundreds of micron large polycrystals; injected in a form of slurry of finely ground powder; fed to an animal ${ }^{61}$; or implanted with tumor cells during inoculation in mice ${ }^{62,63}$. One of their major advantages is that the oxygen sensing is physically decoupled from the local environment. $\mathrm{O}_{2}$ must diffuse into a pore or channel where it interacts with an individual phthalocyanine molecule, which is part of a helical stack. The interaction locally breaks the symmetry of the exchangenarrowed, electron cloud of the stack of phthalocyanine molecules lining the channel, increasing its relaxation rate or linewidth.

Limited mobility of particulates enables repeated measurements of oxygen concentration for an extended time. The higher concentration of unpaired electrons or spin density in comparison with a soluble probe produces higher signal and local sensitivity, although spectroscopy in the absence of imaging gives reduced knowledge of the location of the signal. Spin probe migration and degradation leads to loss of EPR signal intensity. There remain potential biocompatibility concerns with prolonged exposure of particulates to tissue, unless the spin probe is excised. Biocompatible coating of the crystalline probes may overcome some of these problems and enhance their clinical applicability ${ }^{64}$.

\section{Techniques for EPR Imaging $\mathrm{pO}_{2}$}

For imaging, the location in space of a paramagnetic species is encoded using magnetic fields that vary linearly in space, or magnetic field gradients, denoted by a vector along the gradient direction, G. Gradients are designed to alter only local amplitude and not direction 
of the constant magnetic field. The additional magnetic field experienced by a species at position $\mathbf{x}$ in the sample is then $\Delta \mathrm{B}=\mathbf{G} \cdot \mathbf{x}$. Here $\mathbf{x}$ is a three-dimensional spatial coordinate in vector form. Since, from Eq. [3], $\omega_{0}=\gamma_{e} B_{0}, k=\Delta \omega_{0}=\gamma_{e} \Delta B$ this is referred to as frequency encoding. The space of frequencies is referred to as $k$ space. ${ }^{49,65,66}$ Each measurement represents a trajectory in $k$ space. The trajectory for a static gradient is a radial line. In the process of obtaining a $\mathrm{CW}$ spectroscopic image, $B_{0}$ is swept. Fourier transform of the resulting spectra lies along the radial line. This is referred to as a projection, whose angles are defined by the directional part of G. Alternately, a very short pulse of radiofrequency is applied to the sample. This broadband pulse in the presence of a gradient excites all of the spins in the sample. The time trace of the pulse experiment can be mapped to $k$-space directly, as $k=\gamma G T$. It is, thus, a very efficient imaging acquisition strategy. This strategy is referred to as radial imaging and requires filtered backprojection tomographic image reconstruction to recover the images. For oxygen imaging, pulse techniques are used with different detection times after a magnetization inversion to determine the spin-lattice relaxation rate.

A second method for encoding spatial position origin of signal is referred to as phase encoding. Because the relaxation of electrons is very rapid, a variation of the pulse phase encoding technology used in solid state MRI is used. This involves measuring a signal at a the single time point, $t_{\mathrm{p}}$, after a pulse that rotates the magnetization into a plane perpendicular to the magnetic field. Very rapid gradient vector angle and magnitude stepping to cover a 3D Cartesian grid is necessary. Coverage of $k$-space is produced by selection of gradient $k_{\mathrm{ijk}}=\gamma G_{i j k} t_{p}$, one for each $k$-space point. The technique is referred to as single point imaging ${ }^{67}$. It produces images on a Cartesian grid with a much larger number of acquisitions than tomographic imaging. ${ }^{68}$ As a result, it is less efficient than filtered back projection, but freer from artifact imposed by radial $k$-space coverage.

\section{Oxygen Imaging as Longitudinal Relaxation $\left(\mathbf{R}_{1}\right)$ Parametric Imaging}

Oxygen images are derived from three dimensional images of the average longitudinal relaxation rate of the trityl spin probe in each voxel or sub volume that is resolved in the image. This relaxation rate is related to the oxygen concentration or partial pressure through a relevant calibration.

$$
\mathrm{pO}_{2}=a R_{\mathrm{i}}+b \quad[7]
$$

where $i=1$ for longitudinal relaxation and $i=2$ for transverse relaxation. As shown in Fig. 2, the constant, $b$ is far less sensitive to the concentration of spin probe for $R_{1}$ than for $R_{2}$. One of the main advantages of pulse methods, where multiple spatial images are obtained, each at a different delay time relative to a magnetization orientation pulse, is their ability to determine the relaxation rates directly. The 3D spatial images are reconstructed separately. Then in each image the voxel in the same location is selected. Pulse sequences that have a relaxation sensitive component (for example the delay) are used for imaging. This forms a time dependence of the signal for each voxel. Finally, this time dependence is fitted to exponential decay or recovery, and relaxation rate is extracted. The information obtained to reconstruct each image is obtained from different delay times between the inversion pulse 
for $R_{1}$ images, and from the echo time for $R_{2}$ images. These times are so thoroughly interleaved (separated by a microsecond or less), that the reconstructed voxel locations are remarkably stable and reproducible. One of the advantages of the use of highly water soluble trityl spin probes is that their distribution volume is extracellular. Use of $R_{1}$-sensitive pulse sequences virtually eliminates confounding variation from spin probe concentrationdependent effects.

\section{EPR imaging in Cancer Biology}

Several laboratories report various spin probe EPR oxygen measurement techniques, including spectral-spatial continuous wave imaging and localized spectroscopies and pulsebased imaging. ${ }^{69-74}$ Here we describe work in our laboratory focusing on images of tumors in animals - mice, although we have demonstrated both pulse and continuous wave EPR images from rats and rabbits. ${ }^{75,76} \mathrm{We}$ will also describe our ideas for the progression of the technique of spin lattice relaxation (SLR) -based imaging of oxygen tumors to human subjects.

\section{Validation of the Oxymetry in Animals}

Validation of the biologic relevance of oxygen images in animals has required three sets of experiments. We first compared the $\mathrm{pO}_{2}$ from image voxels (volume elements) with point fluorescence quenching measurements using an Oxylite fiberoptic probe ${ }^{77}$. We used a stereotactic needle insertion device to locate the end of the $200 \mu \mathrm{m}$ diameter optical fiber in a FSa mouse fibrosarcoma tumor, and registered it with the EPR oxygen image of the tumor. The Oxylite fiber tip was inserted just after tumor $\mathrm{pO}_{2}$ imaging, inside the imaging resonator/sample holder without disturbing the tumor location. The tumor born by the mouse leg in our resonator with the stereotactically registered fiberoptic probe is shown in Figure 3A. Figure 3B shows examples of $0.7 \mathrm{~mm}$ thick $\mathrm{pO}_{2}$ image slices of a mouse leg bearing a tumor. Two orthogonal planes are shown, with a thick line indicating the path of the fiberoptic Oxylite probe, assuming the fiber to pass from the truly registered entrance straight along the axis of the resonator. The $\mathrm{pO}_{2}$ values measured in the Oxylite tracks as the probe was withdrawn from the tumor and the corresponding $\mathrm{EPR} \mathrm{pO}_{2}$ values from voxels attributed to the track location are shown between the images.

For the second validation, we measured voxel $\mathrm{pO}_{2}$ values within regions of tumors, that were subsequently sampled by a number 12 breast biopsy needle, and compared them with the average levels of hypoxia induced proteins produced by the tumor cells. $\mathrm{pO}_{2}$ images of the tumor were registered with quantitative enzyme-linked immunosorbent assay (ELISA) determinations of the concentration of the hypoxia induced protein Vascular Endothelial Growth Factor from stereotactically localized biopsies from FSa fibrosarcomas. Fig. 4 presents a scatter plot of 17 biopsies, showing typical biological scatter and high statistical significance. About $2 / 3$ of the variation in the protein concentration is associated with the mean $\mathrm{pO}_{2}$, which arises from the image of the volume sampled. Approximately 100 image voxels were localized to each biopsy volume. ${ }^{78}$

Locally administered Tumor Necrosis Factor (TNF), produced by viral vectors carrying genes to promote the cellular synthesis of TNF (referred to as TNFerade) injected directly 
into tumors, has shown dramatic sensitization of tumors to radiation therapy in early human trials. Although this method of treatment of tumors failed a phase 3 trial, there were a number of remarkable cures in phase 2 trials. ${ }^{79}$ This is contradictory to a history of tumor biology showing that hypoxia in tumors creates resistance to radiation therapy. ${ }^{1}$ This prompts inquiry as to the contradictory obliteration of tumor vasculature, which, in principle, would increase tumor hypoxia and increase tumor sensitivity to radiation. EPR $\mathrm{pO}_{2}$ images showed ${ }^{80}$ that tumor $\mathrm{pO}_{2}$ increased after TNF administration. These findings were consistent with the difficulty of chaotic, dysfunctional tumor vasculature to convey the $51 \mathrm{kD}$ TNF protein away from the injection site. Increasing vascular obliteration of intact microvessels, on the other hand, can rid the tumor of TNF. Thus, TNFerade pruned the tumor of chaotic vessels as predicted by Jain et al. ${ }^{81}$ Consistently, tumor sensitization with the anti-angiogenic drug, Sunitinib, which also interferes with tumor angiogenesis, has been shown to be associated with a similarly paradoxical increase in EPR image-based oxygenation from this anti-blood vessel agent. ${ }^{82} \mathrm{EPR} \mathrm{pO} 2$ images provide in situ information defining the molecular biologic response to the microenvironment $\mathrm{pO}_{2}$. EPR imaging is a powerful tool in defining graded molecular biologic response to microenvironment.

The $\mathrm{pO}_{2}$ images that have been obtained with longitudinal relaxation rate pulsed technology have spatial resolution of approximately $1 \mathrm{~mm}$. A major question to be answered is the biological relevance of this resolution. This third validation asks whether or not these images can show, based on the fractions of tumor voxels with $\mathrm{pO}_{2}$, less than a particular threshold, and also, that they can predict outcomes of radiation treatment of tumors of a given size to a dose sufficient to cure $50 \%$ of the tumors. Fig. 5 shows the results of treating two different tumor types, a syngeneic mouse mammary tumor, $\mathrm{MCa} 4$, and a syngeneic fibrosarcoma, $\mathrm{FSa}$, grown in the legs of $\mathrm{C} 3 \mathrm{H}$ mice. The data demonstrate that a threshold of $10 \%$ of voxels with $\mathrm{pO}_{2}$ less than 10 torr for FSa tumors, and $15 \%$ for MCa4 tumors, separates the tumor cure probability. Figure 5 dramatically shows that, for both tumor types, the probability for tumor control was significantly better, a factor of two or more better, for the hypoxic fraction less than the threshold than for the hypoxic fraction larger than the threshold. Therefore, the biomedical relevance of images with the $1 \mathrm{~mm}$ spatial resolution appears strong. ${ }^{83}$

\section{Transient hypoxia can be imaged with $\mathrm{EPR} \mathrm{pO}_{2}$ images}

If tumor hypoxic regions are chronic and unchanging with time, then direction of local therapy such as radiation to such regions would be crucially important. However, if tumor $\mathrm{pO}_{2}$ distributions fluctuate wildly, such images become irrelevant. There is data showing fluctuations in tumor blood flow. This can influence oxygen concentrations. ${ }^{84}$ This may be linked to therapeutic resistance. Dynamic, rapidly acquired images of $\mathrm{pO}_{2}$ are necessary to establish this. Yasui et al. showed that pulsed EPR can monitor fluctuations in oxygen concentrations in mouse models ${ }^{85}$. Oxygen images acquired every 3 minutes for a total of 30 minutes revealed large fluctuations in $\mathrm{pO}_{2}$ in some tumor regions. Redler et al. demonstrated that peripheral regions of tumors with intermediate levels of hypoxia, and which presumably have more intact vasculature, have larger spontaneous $\mathrm{pO}_{2}$ variations then more central regions of FSa fibrosarcomas. ${ }^{86}$ This EPR imaging technique, registered 
with tumor locating MRI, may offer a powerful clinical tool to noninvasively detect variable oxygenation in tumors.

\section{Effectiveness of localizing radiation to regions of the tumor with high hypoxic fraction using EPR pO2 images needs to be tested}

The success noted above in identification of regions within a tumor whose voxels are hypoxic, and showing that large fractions of these voxels induce resistance to a $50 \%$ tumor control dose, leads to the question: Does treating only these regions of tumors with extra 'boost' dose increase the tumor cure? This needs to be demonstrated in animal models prior to human applications. Figs. 6A and 6B show an hypoxic region defined in a mouse MCa4 breast tumor, and a spherical volume for radiation boost treatment. This boost would be added to a 50\% control dose given to the entire tumor. Figs. 6C-E show an XRAD225CX system to precisely deliver the boost dose to the indicated region. A trial comparing the tumor control using such a boost with treatment to a well oxygenated shell of similar volume (an "anti-boost") is underway. ${ }^{87}$

\section{Summary}

We have brought the technology for the EPR imaging of tumor $\mathrm{pO}_{2}$ to a point where quantitative images of oxygen in the tissues and tumors of living animals, with 1-2 torr $\mathrm{pO}_{2}$ resolution and $1 \mathrm{~mm}$ spatial resolution, can be obtained in 10 minutes or faster. We have shown the biologic relevance of the oxygen images and demonstrated that they predict cure from radiation therapy. Rapid acquisition of $\mathrm{EPR} \mathrm{pO}_{2}$ images will validate the relevance of transient hypoxia. We are in the process of validation of the potential use of EPR oxygen images in guiding radiation to resistant portions of animal tumors. A path to human applications may be possible.

\section{Acknowledgments}

This work was supported by NIH grants P41 EB002034 and R01 CA098575

\section{References}

1. Thomlinson RH, Gray LH. The histological structure of some human lung cancers and the possible implications for radiotherapy. Br J Radiol. 1955; 9:539-563.

2. Kennedy KA, Teicher BA, Rockwell S, Sartorelli AC. The hypoxic tumor cell: a target for selective cancer chemotherapy. Biochem Pharmacol. 1980; 29:1-8. [PubMed: 6987986]

3. Teicher BA. Hypoxia and drug resistance. Cancer Metastasis Rev. 1994; 13:139-168. [PubMed: 7923547]

4. Henk JM, Kunkler PB, Smith CW. Radiotherapy and hyperbaric oxygen in head and neck cancer. Final report of first controlled clinical trial. Lancet. 1977; 2:101-103. [PubMed: 69191]

5. Henk JM, Smith CW. Radiotherapy and hyperbaric oxygen in head and neck cancer. Interim report of second clinical trial. Lancet. 1977; 2:104-105. [PubMed: 69192]

6. Rontgen WC. On a New Kind of Rays. Science. 1896; 3:227-231.

7. Cormack AM. Representation of a Function by Its Line Integrals with Some Radiological Applications. J Appl Phys. 1963; 34:2722-\&.

8. Lauterbur PC. Image Formation by Induced Local Interactions - Examples Employing Nuclear Magnetic-Resonance. Nature. 1973; 242:190-191. 
9. Condon, EU.; Shortley, GH. The Theory of Atomic Spectra. Cambridge University Press; 1935.

10. Lauterbur PC, Levin DN, Marr RB. Theory and simulation of NMR spectroscopic imaging and field plotting by projection reconstruction involving an intrinsic frequency dimension. J Magn Reson. 1984; 59:536-541.

11. Maltempo MM. Differentiation of spectral and spatial components in EPR imaging using 2-D image reconstruction algorithms. J Magn Reson. 1986; 69:156-161.

12. Eastman PE, Kooser RG, Pas MR, Freed JH. Studies of Heisenberg spin exchange in ESR spectra I. Linewidth and saturation effects. J Chem Phys. 1969; 54:2690.

13. Molin, YN.; Salikhov, KM.; Zamaraev, KI. Spin Exchange: Principles and Applications in Chemistry and Biology. Berlin: Springer-Verlag; 1980.

14. Swartz, HM.; Glockner, JF. Measurements of Oxygen by EPRI and EPRS. Boca Raton, FL: CRC Press, Inc.; 1991.

15. Whalen WJ, Riley J, Nair P. A microelectrode for measuring intracellular pO2. J Appl Physiol. 1967; 23:798-801. [PubMed: 6061398]

16. Harrison, DK. Physiological oxygen measurements using oxygen electrodes. In: Wilson, DFESYBJPA., editor. Oxygen Transport to Tissue Volume Xxiii: Oxygen Measurements in the 21st Century: Basic Techniques and Clinical Relevance. 2003. p. 163-167.

17. Griffiths JR, Robinson SP. The OxyLite: a fibre-optic oxygen sensor. British Journal of Radiology. 1999; 72:627-630. [PubMed: 10624317]

18. Krause BJ, Beck R, Souvatzoglou M, Piert M. PET and PET/CT studies of tumor tissue oxygenation. Quarterly Journal of Nuclear Medicine and Molecular Imaging. 2006; 50:28-43. [PubMed: 16557202]

19. Ogawa S, Lee TM. MAGNETIC-RESONANCE-IMAGING OF BLOOD-VESSELS AT HIGH FIELDS - INVIVO AND INVITRO MEASUREMENTS AND IMAGE SIMULATION. Magnet Reson Med. 1990; 16:9-18.

20. Ogawa S, Lee TM, Kay AR, Tank DW. BRAIN MAGNETIC-RESONANCE-IMAGING WITH CONTRAST DEPENDENT ON BLOOD OXYGENATION. Proc Natl Acad Sci USA. 1990; 87:9868-9872. [PubMed: 2124706]

21. Rumsey WL, Vanderkooi JM, Wilson DF. Imaging of Phosphorescence - a Novel Method for Measuring Oxygen Distribution in Perfused Tissue. Science. 1988; 241:1649-1651. [PubMed: 3420417]

22. Busse LJ, Pratt RG, Thomas SR. DECONVOLUTION OF CHEMICAL-SHIFT SPECTRA IN TWO-DIMENSIONAL OR 3-DIMENSIONAL F-19 MR IMAGING. Journal of Computer Assisted Tomography. 1988; 12:824-835. [PubMed: 3170845]

23. Hunjan S, Zhao D, Constantinescu A, Hahn EW, Antich PP, Mason RP. Tumor oximetry: demonstration of an enhanced dynamic mapping procedure using fluorine-19 echo planar magnetic resonance imaging in the Dunning prostate R3327-AT1 rat tumor. Int J Radiat Oncol Biol Phys. 2001; 49:1097-1108. [PubMed: 11240252]

24. Efimova OV, Caia GL, Sun ZQ, et al. Standard-based method for proton-electron double resonance imaging of oxygen. J Magn Reson. 2011; 212:197-203. [PubMed: 21807539]

25. Schweiger, A.; Jeschke, G. Principles of Pulse Electron Paramagnetic Resonance. Oxford University Press; 2001.

26. Slichter, CP. Principles of Magnetic Resonance. New York: Springer-Verlag; 1996.

27. Abragam, A.; Bleaney, B. EPR of Transition Ions. Oxford: Clarendon; 1970.

28. Atherton, NM. Principles of Electron Spin Resonance. Chichester: Ellis Horwood Ltd; 1993.

29. Dirac, PAM. Principles of Quantum Mechanics. 4th (paperback). Oxford University Press; 1982.

30. Abragam, A. Principles of Nuclear Magnetism. Oxford: Oxford University; 1961.

31. Ahmad, R.; Khan, M.; Vikram, D.; Bratasz, A.; Kuppusamy, P. EPR Oximetry: Method and Application. In: Das, D., editor. Methods in Redox Signaling. Mary Ann Liebert, Inc.; 2010.

32. Tatum JL, Kelloff GJ, Gillies RJ, et al. Hypoxia: Importance in tumor biology, noninvasive measurement by imaging, and value of its measurement in the management of cancer therapy. Int $\mathbf{J}$ Radiat Biol. 2006; 82:699-757. [PubMed: 17118889] 
33. Khan N, Williams BB, Hou H, Li H, Swartz HM. Repetitive tissue pO(2) measurements by electron paramagnetic resonance oximetry: Current status and future potential for experimental and clinical studies. Antioxidants \& Redox Signaling. 2007; 9:1169-1182. [PubMed: 17536960]

34. Backer JM, Budker VG, Eremenko SI, Molin YN. Detection of the kinetics of biochemical reactions with oxygen using exchange broadening in the ESR spectra of nitroxide radicals. Biochim Biophys Acta. 1977; 460:152-156. [PubMed: 192284]

35. Popp CA, Hyde JS. Effects of Oxygen on Electron-Paramagnetic-Resonance of Nitroxide SpinLabel Probes of Model Membranes. J Magn Reson. 1981; 43:249-258.

36. Swartz HM, Clarkson RB. The measurement of oxygen in vivo using EPR techniques. Physics in Medicine and Biology. 1998; 43:1957-1975. [PubMed: 9703059]

37. Halpern HJ, Yu C, Peric M, Barth E, Grdina DJ, Teicher BA. Oxymetry Deep in Tissues with Low-Frequency Electron-Paramagnetic-Resonance. Proc Natl Acad Sci USA. 1994; 91:1304713051. [PubMed: 7809170]

38. Zweier JL, Thompsongorman S, Kuppusamy P. Measurement of Oxygen Concentrations in the Intact Beating Heart Using Electron-Paramagnetic Resonance Spectroscopy - a Technique for Measuring Oxygen Concentrations Insitu. J Bioenerg Biomembr. 1991; 23:855-871. [PubMed: 1663949]

39. Kuppusamy P, Chzhan M, Vij K, et al. 3-Dimensional Spectral Spatial EPR Imaging of FreeRadicals in the Heart - a Technique for Imaging Tissue Metabolism and Oxygenation. Proc Natl Acad Sci USA. 1994; 91:3388-3392. [PubMed: 8159757]

40. Mailer C, Robinson BH, Williams BB, Halpern HJ. Spectral fitting: The extraction of crucial information from a spectrum and a spectral image. Magnet Reson Med. 2003; 49:1175-1180.

41. Robinson BH, Mailer C, Reese AW. Linewidth analysis of spin labels in liquids - I. Theory and data analysis. J Magn Reson. 1999; 138:199-209. [PubMed: 10341123]

42. Robinson BH, Mailer C, Reese AW. Linewidth analysis of spin labels in liquids - I. Experimental. J Magn Reson. 1999; 138:210-219. [PubMed: 10341124]

43. Swartz HM, Khan N, Buckey J, et al. Clinical applications of EPR: overview and perspectives. NMR Biomed. 2004; 17:335-351. [PubMed: 15366033]

44. Wilson, JD.; Braunwald, E.; Isselbacher, KJ., et al. Harrison's Principles of Internal Medicine. 12. New York: McGraw-Hill; 1991.

45. Eaton, SS.; Eaton, GR. Biological Magnetic Resonance. New York: Kluwer Academic/Plenum Publishers; 2000. Relaxation Times of Organic Radicals and Transition Metal Ions.

46. Longo, DL.; Fauci, AS.; Kasper, DL.; Hauser, SL.; Jameson, JL.; Loscalzo, J., editors. Harrison's Principles of Internal Medicine. 18. New York, NY: McGraw Hill; 2011.

47. Halpern HJ, Spencer DP, Vanpolen J, et al. Imaging Radio-Frequency Electron-Spin-Resonance Spectrometer with High-Resolution and Sensitivity for Invivo Measurements. Rev Sci Instrum. 1989; 60:1040-1050.

48. Bottomley PA, Andrew ER. RF magnetic field penetration, phase shift and power dissipation in biological tissue: implications for NMR imaging. Phys Med Biol. 1978; 23:630-643. [PubMed: 704667]

49. Mailer C, Sundramoorthy SV, Pelizzari CA, Halpern HJ. Spin echo spectroscopic electron paramagnetic resonance imaging. Magn Reson Med. 2006; 55:904-912. [PubMed: 16526015]

50. Epel B, Bowman MK, Mailer C, Halpern HJ. Absolute oxygen R1 imaging in vivo with pulse electron paramagnetic resonance. Magn Reson Med. 2014; 72:362-368. [PubMed: 24006331]

51. Dunn, JW.; Swartz, HM., editors. Oxygen Transport to Tissue XXIV. New York: Kluwer Academic/Plenum Publishers; 2003.

52. Berliner, LJ., editor. In Vivo EPR (ESR): Theory \& Applications. New York: Kluwer Academic; 2003.

53. Berliner LJ, Fujii H. Magnetic-Resonance Imaging of Biological Specimens by ElectronParamagnetic Resonance of Nitroxide Spin Labels. Science. 1985; 227:517-519. [PubMed: 2981437]

54. Biller JR, Meyer V, Elajaili H, et al. Relaxation times and line widths of isotopically-substituted nitroxides in aqueous solution at X-band. J Magn Reson. 2011; 212:370-377. [PubMed: 21843961] 
55. Ardenkjaer-Larsen JH, Laursen I, Leunbach I, et al. EPR and DNP properties of certain novel single electron contrast agents intended for oximetric imaging. J Magn Reson. 1998; 133:1-12. [PubMed: 9654463]

56. Pandian RP, Parinandi NL, Ilangovan G, Zweier JL, Kuppusamy P. Novel particulate spin probe for targeted determination of oxygen in cells and tissues. Free Radical Biology and Medicine. 2003; 35:1138-1148. [PubMed: 14572616]

57. Williams BB, al Hallaq H, Chandramouli GV, et al. Imaging spin probe distribution in the tumor of a living mouse with $250 \mathrm{MHz}$ EPR: correlation with BOLD MRI. Magn Reson Med. 2002; 47:634-638. [PubMed: 11948723]

58. Matsumoto K, English S, Yoo J, et al. Pharmacokinetics of a triarylmethyl-type paramagnetic spin probe used in EPR oximetry. Magnet Reson Med. 2004; 52:885-892.

59. Krishna MC, English S, Yamada K, et al. Overhauser enhanced magnetic resonance imaging for tumor oximetry: Coregistration of tumor anatomy and tissue oxygen concentration. Proc Natl Acad Sci USA. 2002; 99:2216-2221. [PubMed: 11854518]

60. Liu KJ, Gast P, Moussavi M, et al. Lithium Phthalocyanine - a Probe for Electron-ParamagneticResonance Oximetry in Viable Biological-Systems. Proc Natl Acad Sci USA. 1993; 90:54385442. [PubMed: 8390665]

61. He GL, Shankar RA, Chzhan M, Samouilov A, Kuppusamy P, Zweier JL. Noninvasive measurement of anatomic structure and intraluminal oxygenation in the gastrointestinal tract of living mice with spatial and spectral EPR imaging. Proc Natl Acad Sci USA. 1999; 96:4586-4591. [PubMed: 10200306]

62. Ilangovan G, Bratasz A, Kuppusamy P. Non-invasive measurement of tumor oxygenation using embedded microparticulate EPR spin probe. Oxygen Transport to Tissue Xxvi. 2005; 566:67-73.

63. Ilangovan G, Bratasz A, Li H, Schmalbrock P, Zweier JL, Kuppusamy P. In vivo measurement and imaging of tumor oxygenation using coembedded paramagnetic particulates. Magn Reson Med. 2004; 52:650-657. [PubMed: 15334586]

64. Meenakshisundaram G, Pandian RP, Eteshola E, Lee SC, Kuppusamy P. A paramagnetic implant containing lithium naphthalocyanine microcrystals for high-resolution biological oximetry. J Magn Reson. 2010; 203:185-189. [PubMed: 20006529]

65. Epel, B.; Halpern, HJ. Electron paramagnetic resonance oxygen imaging in vivo. In: Gilbert, BC.; Chechik, V.; Murphy, DM., editors. Electron Paramagnetic Resonance. Cambridge, UK: RSC Publishing; 2012.

66. Epel B, Sundramoorthy SV, Mailer C, Halpern HJ. A versatile high speed 250-MHz pulse imager for biomedical applications. Concepts in Magnetic Resonance Part B: Magnetic Resonance Engineering. 2008; 33B:163-176. [PubMed: 19924261]

67. Subramanian S, Devasahayam N, Murugesan R, et al. Single-point (constant-time) imaging in radiofrequency Fourier transform electron paramagnetic resonance. Magn Reson Med. 2002; 48:370-379. [PubMed: 12210946]

68. Epel B, Halpern HJ. Comparison of pulse sequences for R-based electron paramagnetic resonance oxygen imaging. J Magn Reson. 2015; 254:56-61. [PubMed: 25828242]

69. Khan, N.; Hou, H.; Hein, P., et al. Black Magic and EPR Oxymetry: From Lab to Initial Clinical Trials. New York: Plenum Publishers; 2005.

70. Salikhov I, Walczak T, Lesniewski P, et al. EPR spectrometer for clinical applications. Magn Reson Med. 2005; 54:1317-1320. [PubMed: 16193470]

71. Khan N, Williams BB, Hou H, Li H, Swartz HM. Repetitive tissue pO2 measurements by electron paramagnetic resonance oximetry: current status and future potential for experimental and clinical studies. Antioxid Redox Signal. 2007; 9:1169-1182. [PubMed: 17536960]

72. Williams BB, Khan N, Zaki B, Hartford A, Ernstoff MS, Swartz HM. Clinical electron paramagnetic resonance (EPR) oximetry using India ink. Adv Exp Med Biol. 2010; 662:149-156. [PubMed: 20204785]

73. Ellis SJ, Velayutham M, Velan SS, et al. EPR oxygen mapping (EPROM) of engineered cartilage grown in a hollow-fiber bioreactor. Magn Reson Med. 2001; 46:819-826. [PubMed: 11590660]

74. Zweier JL, Chzhan M, Wang PH, Kuppusamy P. Spatial and Spectral-Spatial EPR Imaging Of Free Radicals and Oxygen In the Heart. Research On Chemical Intermediates. 1996; 22:615-624. 
75. Epel B, Haney CR, Hleihel D, Wardrip C, Barth ED, Halpern HJ. Electron paramagnetic resonance oxygen imaging of a rabbit tumor using localized spin probe delivery. Med Phys. 2010; 37:25532559. [PubMed: 20632567]

76. Epel B, Sundramoorthy SV, Barth ED, Mailer C, Halpern HJ. Comparison of $250 \mathrm{MHz}$ electron spin echo and continuous wave oxygen EPR imaging methods for in vivo applications. Med Phys. 2011; 38:2045-2052. [PubMed: 21626937]

77. Elas M. Electron Paramagnetic Resonance Oxygen Images Correlate Spatially and Quantitatively with Oxylite Oxygen Measurements. Clinical Cancer Research. 2006; 12:4209-4217. [PubMed: 16857793]

78. Elas M, Hleihel D, Barth ED, et al. Where it's at really matters: in situ in vivo vascular endothelial growth factor spatially correlates with electron paramagnetic resonance $\mathrm{pO} 2$ images in tumors of living mice. Mol Imaging Biol. 2011; 13:1107-1113. [PubMed: 20960236]

79. Mundt AJ, Vijayakumar S, Nemunaitis J, et al. A Phase I trial of TNFerade biologic in patients with soft tissue sarcoma in the extremities. Clin Cancer Res. 2004; 10:5747-5753. [PubMed: 15355902]

80. Haney CR, Parasca AD, Fan X, et al. Characterization of response to radiation mediated gene therapy by means of multimodality imaging. Magn Reson Med. 2009; 62:348-356. [PubMed: 19449382]

81. Carmeliet P, Jain RK. Molecular mechanisms and clinical applications of angiogenesis. Nature. 2011; 473:298-307. [PubMed: 21593862]

82. Matsumoto S, Batra S, Saito K, et al. Antiangiogenic agent sunitinib transiently increases tumor oxygenation and suppresses cycling hypoxia. Cancer Res. 2011; 71:6350-6359. [PubMed: 21878530]

83. Elas M, Magwood JM, Butler B, et al. EPR oxygen images predict tumor control by a $50 \%$ tumor control radiation dose. Cancer Res. 2013; 73:5328-5335. [PubMed: 23861469]

84. Brown JM. Evidence for Acutely Hypoxic Cells in Mouse-Tumors, and a Possible Mechanism of Re-Oxygenation. Br J Radiol. 1979; 52:650-656. [PubMed: 486895]

85. Yasui H, Matsumoto S, Devasahayam N, et al. Low-Field Magnetic Resonance Imaging to Visualize Chronic and Cycling Hypoxia in Tumor-Bearing Mice. Cancer Res. 2010; 70:64276436. [PubMed: 20647318]

86. Redler G, Epel B, Halpern HJ. EPR image based oxygen movies for transient hypoxia. Adv Exp Med Biol. 2014; 812:127-133. [PubMed: 24729224]

87. Epel B, Redler G, Pelizzari C, Tormyshev VM, Halpern HJ. Approaching oxygen-guided intensitymodulated radiation therapy. Adv Exp Med Biol. 2015 in Press:in Press. 


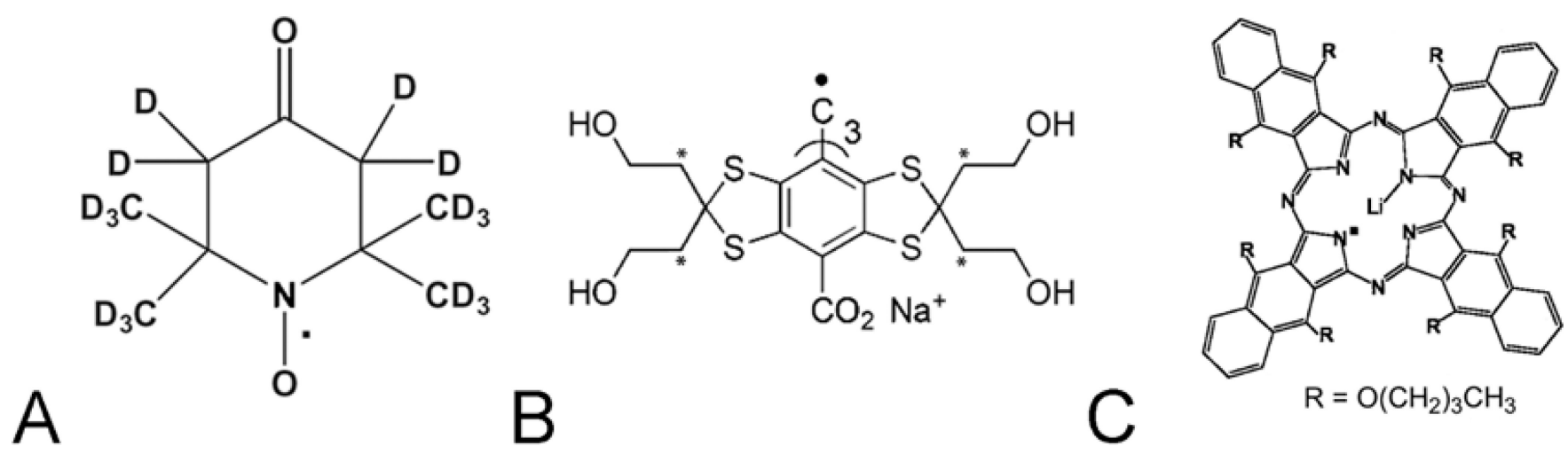

Figure 1.

Chemical structures of typical spin probes : A. water soluble fully deuterated 6-member ring nitroxide; B. water soluble OX071 trityl, the deuterated methylene groups are marked with *; C. particulate LiNc-BuO spin probe. 

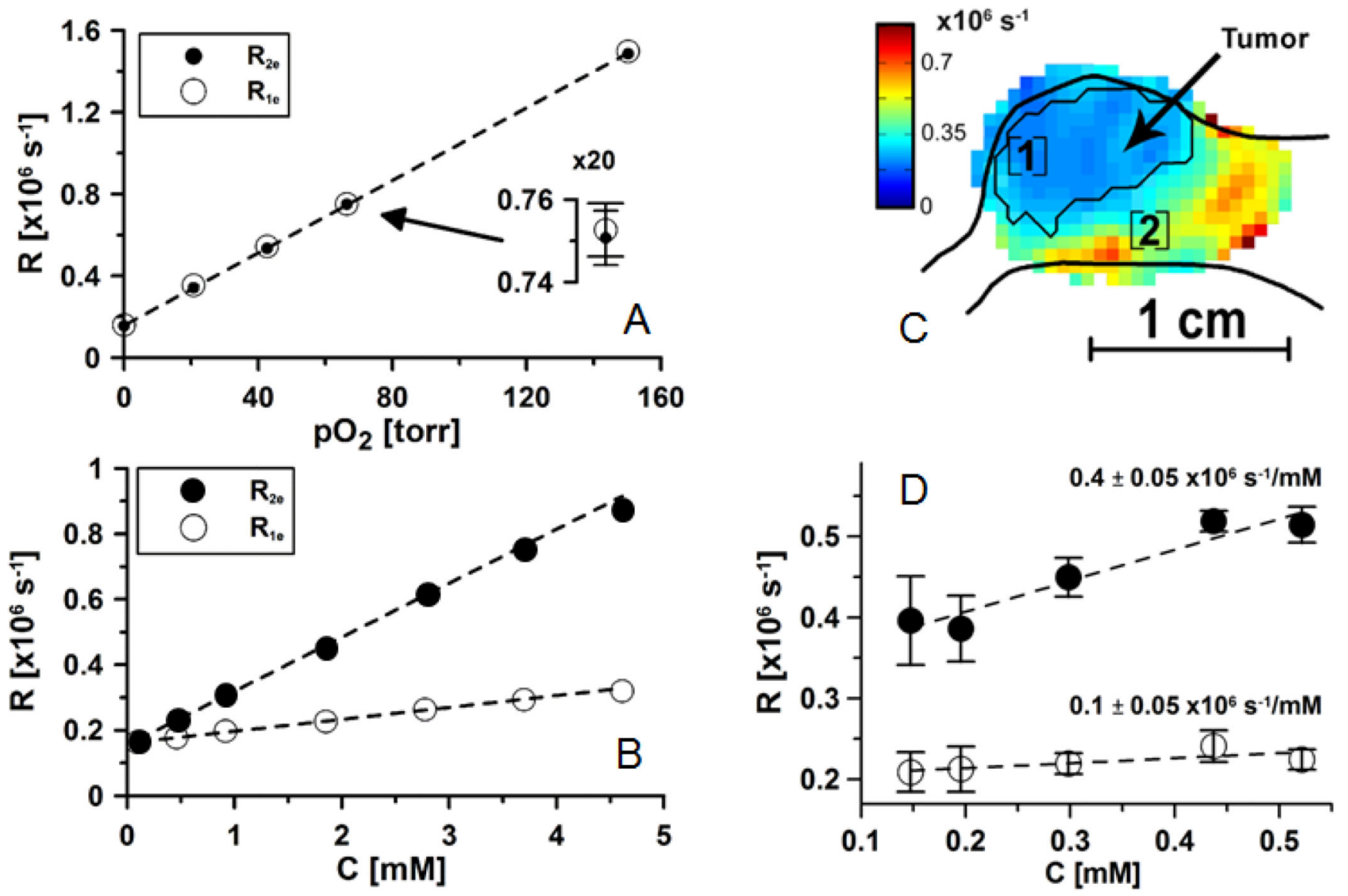

Figure 2.

A and B - relaxation rates of $\mathrm{OX} 063$ dissolved in saline at $37^{\circ} \mathrm{C}$. The range of the measured spin probe concentrations is considerably larger than the one observed in vivo. C. Relaxation rate image. D. Relaxation rates in position marked as [1] in Fig. C. Rates are measured as a function of spin probe concentration while spin probe is infused at different rates, from low to high. The slopes of concentration dependence are given in the plot. 

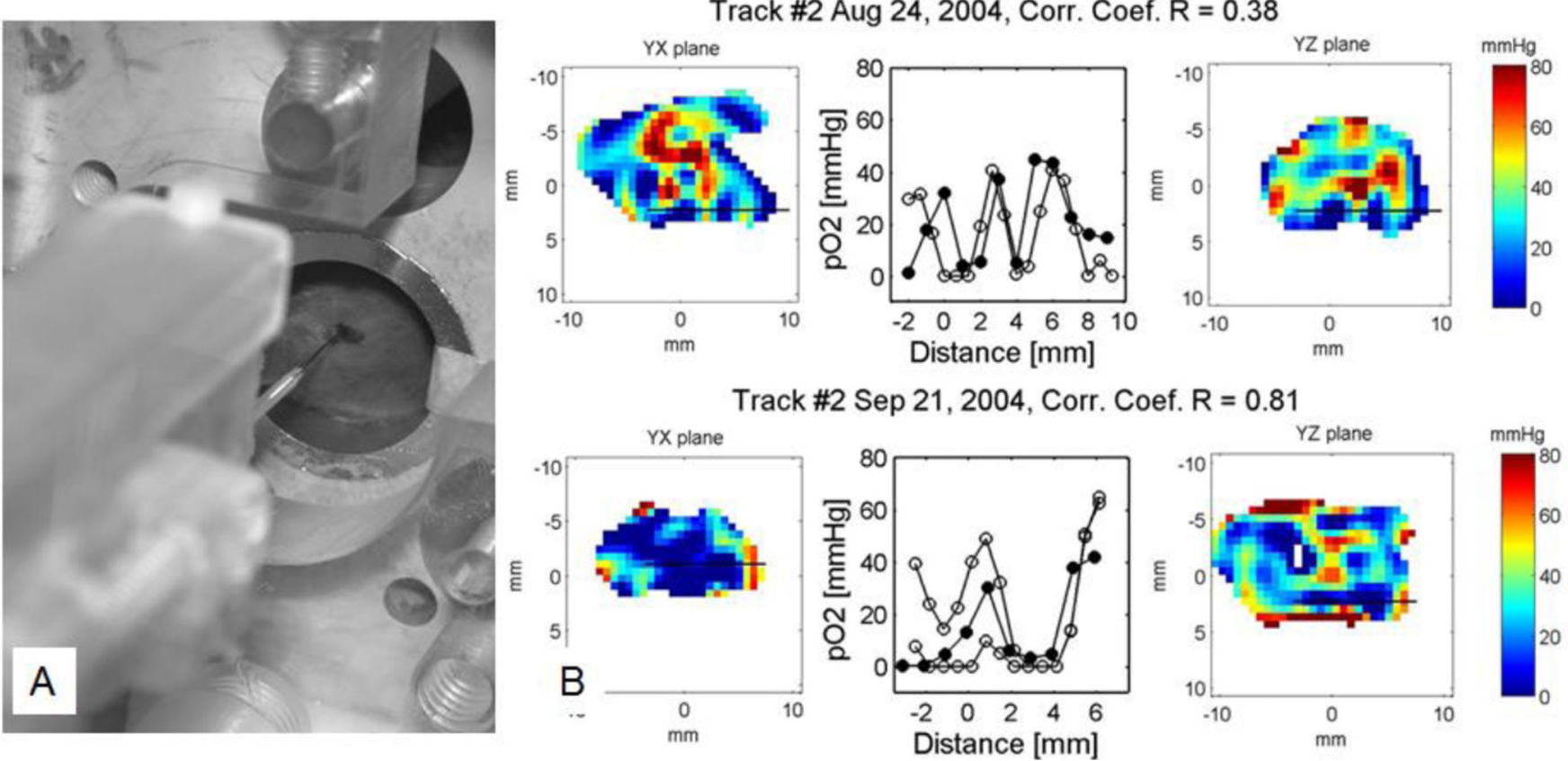

Figure 3.

A. Leg born mouse tumor in an $\mathrm{EPR} \mathrm{pO}_{2}$ imager with an Oxylite fiberoptic $\mathrm{pO}_{2}$ probe being launched into a tumor from a stereotactic frame. B. Two examples of the correlation between EPR and fiberoptic $\mathrm{pO}_{2}$ data. Left and right panels: Sagittal and coronal slices showing tumor $\mathrm{pO}_{2}$. The assumed probe track is shown as a black line in the images. Middle panel: Plot of the oxygen values from the Oxylite ${ }^{\mathrm{TM}}$ track (filled circles) and $\mathrm{pO}_{2}$ image (open circles). The remarkable correlation between these values was observed. 


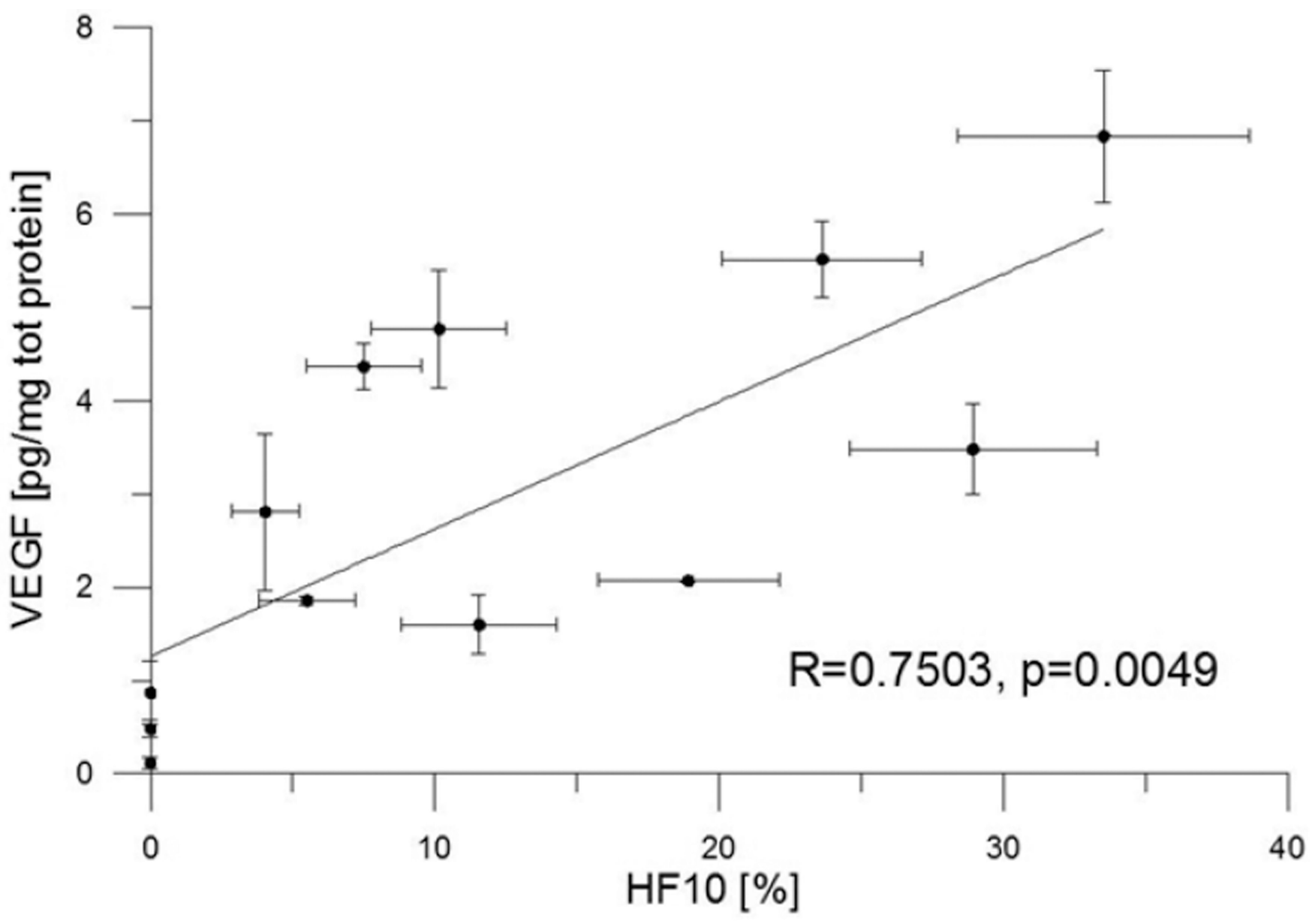

Figure 4.

Correlation between hypoxia and VEGF content. Fraction of volumes in the biopsy with $\mathrm{pO}_{2}$ less than 10 torr, HF10 from the EPR oxygen image vs VEGF concentration in picograms per microgram of total protein. 

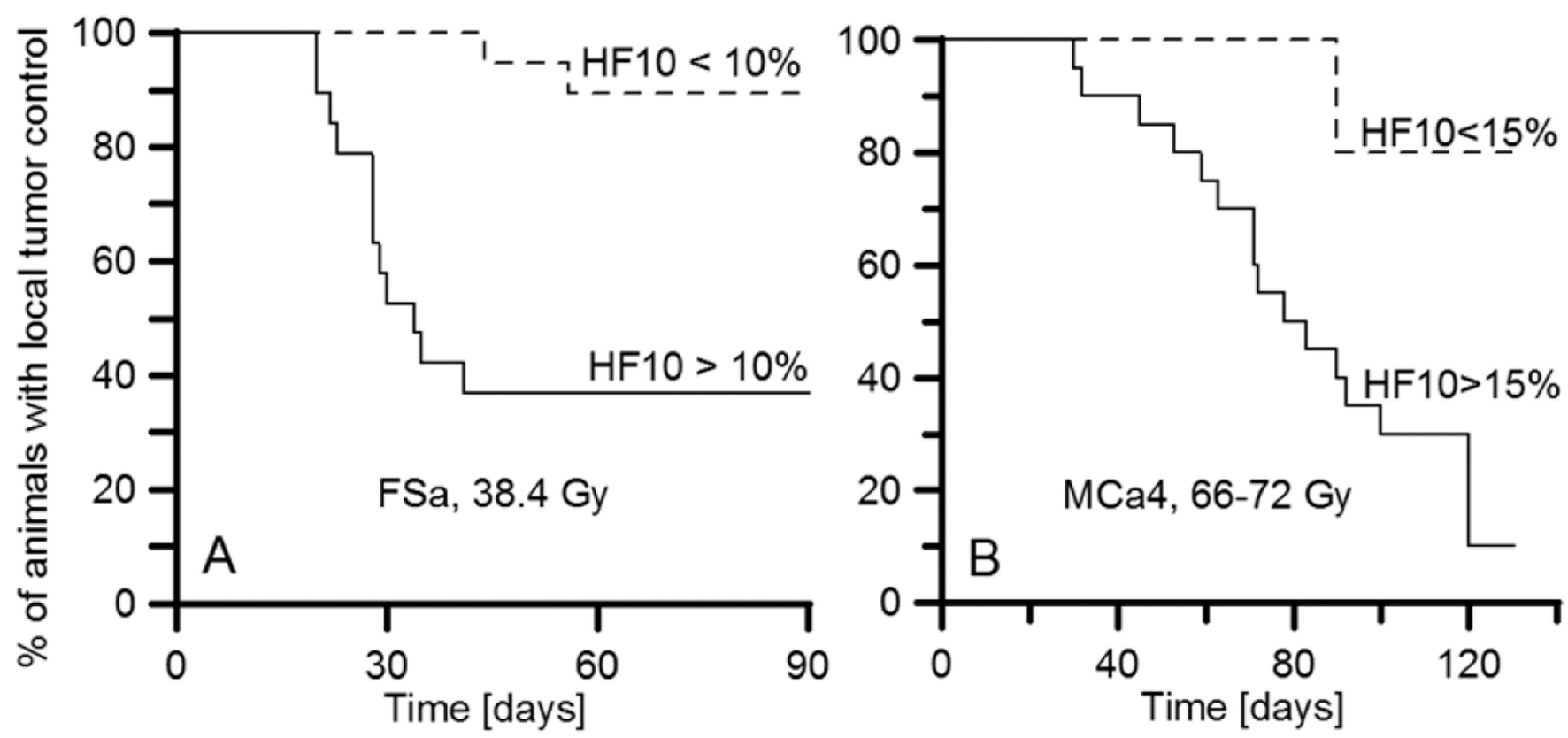

Figure 5.

The outcome of a single X-ray dose treatment. Kaplan-Meier plots for two groups of animals separated according to their tumor hypoxic fraction (HF10), a percent of voxels in the $\mathrm{pO}_{2}$ image with $\mathrm{pO}_{2}$ below 10 torr. A. FSA tumors treated with $33.8 \mathrm{~Gy}$. Wilcoxon test shows that $\mathrm{HF} 10>10 \%$ threshold is a significant predictor of tumor failure $(\mathrm{p}=0.0138)$. B. MCa4 tumors treated with a single dose in the range of 66-72 Gy. Wilcoxon test shows that HF10 $>15 \%$ threshold is a significant predictor of tumor failure $(\mathrm{p}=0.0193)$. 

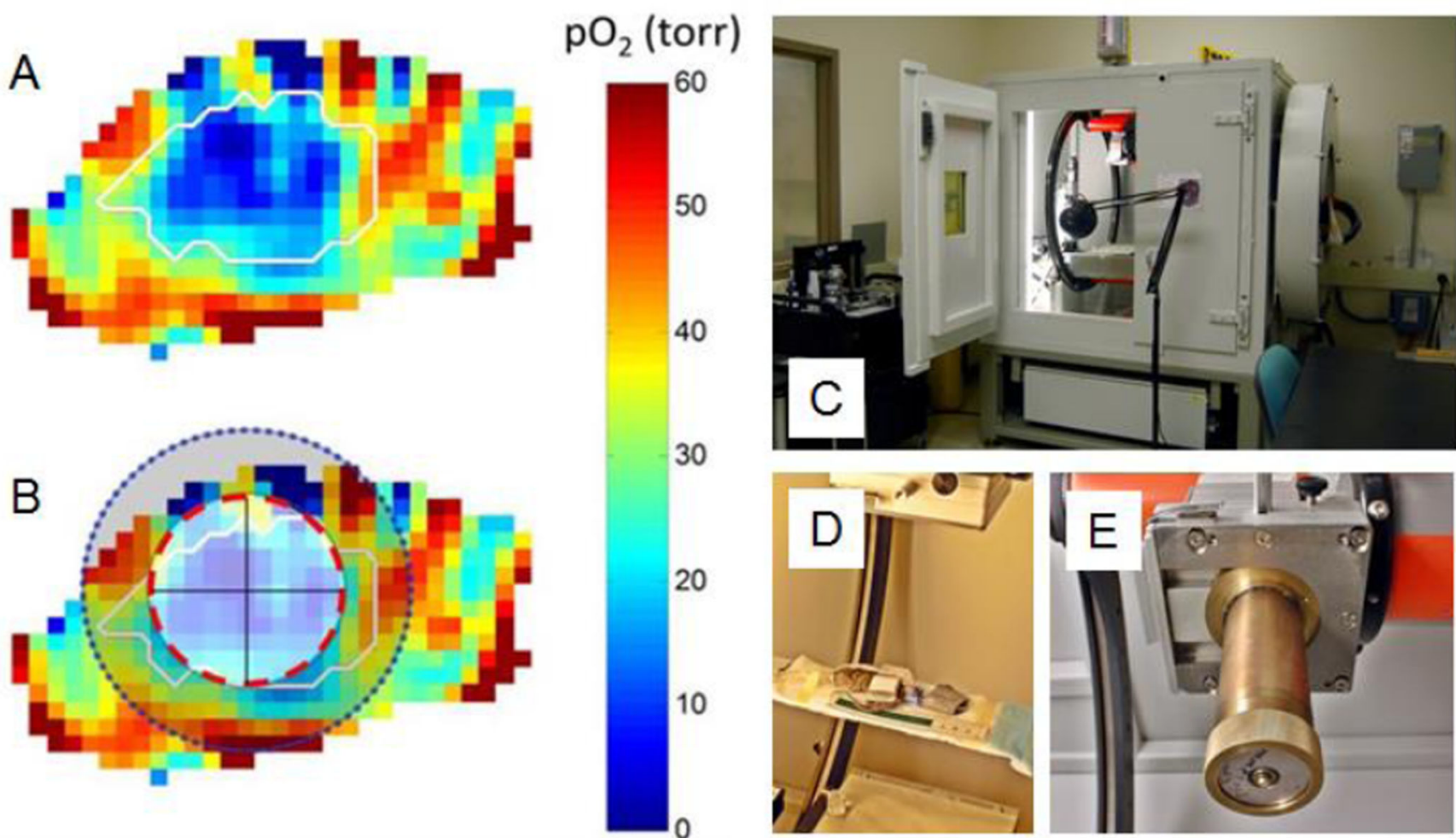

Figure 6.

EPR oxygen image of the leg tumor. A. Oxygen map with tumor contour transferred from the registered MRI image. B. Boost (red line) and anti-boost (shaded area) as determined by the boost planning software. C. D. and E. XRad225Cx small animal cone beam CT imager and irradiator. D. Animal bed. E. Collimator. 